\title{
SPIFI: a direct-detection imaging spectrometer for submillimeter wavelengths
}

\author{
C. Matt Bradford, Gordon J. Stacey, Mark R. Swain, Thomas Nikola, \\ Alberto D. Bolatto, James M. Jackson, Maureen L. Savage, Jacqueline A. Davidson, \\ and Peter A. R. Ade
}

\begin{abstract}
The South Pole Imaging Fabry-Perot Interferometer (SPIFI) is the first instrument of its kind-a direct-detection imaging spectrometer for astronomy in the submillimeter band. SPIFI's focal plane is a square array of 25 silicon bolometers cooled to $60 \mathrm{mK}$; the spectrometer consists of two cryogenic scanning Fabry-Perot interferometers in series with a $60-\mathrm{mK}$ bandpass filter. The instrument operates in the short submillimeter windows $(350$ and $450 \mu \mathrm{m})$ available from the ground, with spectral resolving power selectable between 500 and 10,000. At present, SPIFI's sensitivity is within a factor of 1.5-3 of the photon background limit, comparable with the best heterodyne spectrometers. The instrument's large bandwidth and mapping capability provide substantial advantages for specific astrophysical projects, including deep extragalactic observations. We present the motivation for and design of SPIFI and its operational characteristics on the telescope. (C) 2002 Optical Society of America
\end{abstract}

OCIS codes: $\quad 350.1260,350.1270,050.2230,300.6270,040.6040$.

\section{Introduction}

Spectroscopy at submillimeter frequencies $(\lambda \sim 100$ $\mu \mathrm{m}-1 \mathrm{~mm})$ provides an unique probe of the interstellar medium of our galaxy and external galaxies. The much longer wavelength ensures that dust extinction is completely negligible relative to the nearand mid-IR lines observable from the ground. Yet unlike the typical millimeter- or centimeter-wave molecular transitions, most of the submillimeter lines

When this research was performed, C. M. Bradford, G. J. Stacey, M. R. Swain, and T. Nikola were with the Department of Astronomy, Cornell University, Ithaca, New York 14853. A. D. Bolatto and J. M. Jackson were with the Institute for Astrophysical Research, Boston University, Boston, Massachusetts 02215. M. L. Savage and J. A. Davidson were with the Universities Space Research Association Stratospheric Observatory for Infrared Astronomy, NASA Ames Research Center, Moffet Field, California 94035. P. A. R. Ade was with the Department of Physics and Astronomy, University of Wales, 5, The Parade, Cardiff CF24 3YB, UK. C. M. Bradford (bradford@submm.caltech.edu) is now with the Department of Physics, California Institute of Technology, Pasadena, California 91125. A. D. Bollato is now with the Radio Astronomy Laboratory, University of California, Berkeley, Berkeley, California 94720.

Received 18 October 2001; revised manuscript received 9 January 2002.

0003-6935/02/132561-14\$15.00/0

(C) 2002 Optical Society of America arise only in excited regions. Study of the excited gas is not confused by cool molecular material nearby or along the line of sight. Because submillimeter lines include rotational transitions of warm molecular gas as well as fine-structure transitions of atomic and ionized species, the suite of lines makes an excellent tool for study of the ionized gas and molecular gas interaction zone that results when new stars disrupt the environment of their birth.

From the instrumentation standpoint, the submillimeter band is one of the final frontiers of observational astronomy. As the regime in which direct-detection and heterodyne techniques converge, instrumentation performance has been improving rapidly during the past two decades. Recently, heterodyne receivers have advanced to higher frequencies, become more sensitive, and are beginning to be assembled in small arrays. ${ }^{1,2}$ These receivers have been used successfully to observe submillimeter spectral lines in a variety of galactic and a few extragalactic sources over the past 10-20 years. $^{3-7}$ The advantages of these receivers are that spectral resolution can be made arbitrarily high, and, because they preserve the phase of the radiation field, coherent receivers can be used in interferometry experiments to obtain high angular resolution. The preservation of phase, however, requires amplification of the signal before detection and results in an uncertainty in photon occupation number (intensity) 
of one photon per unit bandwidth per second. When expressed as a temperature of a radiation field that would produce the same noise, this quantum noise is $T_{\mathrm{RX} \mathrm{QN}}(S S B)=h v / k_{B} \cdot{ }^{8-10}$

In the short submillimeter bands, the quantum noise becomes comparable to the fundamental noise because of the fluctuations in the arrival rates of photons: $T_{\mathrm{RX}, \mathrm{PN}} \sim 100-200 \mathrm{~K}$ (temperature times emissivity), a fundamental limit for any detection process (see Section 4). Thus a coherent system is always less sensitive than a system subject only to the photon background noise, with the difference especially significant under low-background conditions such as airborne or spaceborne observatories.

Heterodyne systems are also limited in bandwidth, especially at the higher frequencies where the fractional bandwidth of a fixed spectrometer backend is smaller. At present, 1.5-GHz backends are the largest used astronomically, ${ }^{1}$ which results in a velocity coverage of only $560 \mathrm{~km} \mathrm{~s}^{-1}$ at $810 \mathrm{GHz}$. Although much larger bandwidth systems are under development, this is currently a serious limitation for extragalactic observations where linewidths are approximately a few times $100 \mathrm{~km} \mathrm{~s}^{-1}$. Often multiple spectral setups are required to observe the full line profile. ${ }^{11}$

In contrast, a direct-detection spectrometer can be background limited and have a large bandwidth. Low photon energies prohibit the photon-counting detectors of the mid-IR and far IR, ${ }^{12}$ but bolometers have proven to be effective submillimeter-wave detectors. In continuum instruments, bolometers have approached background-limited performance with single pixels and, recently, in large arrays such as those in the SCUBA (Submillimeter CommonUser Bolometer Array) camera in service at the James Clerk Maxwell Telescope (JCMT).13,14 A background-limited spectrometer is naturally more difficult than a continuum instrument; spectroscopy requires a large cryogenic interference path length, and, because even 4-K surfaces emit substantially in the submillimeter bands, careful filtration is essential. Furthermore, the background power and corresponding photon noise on the detectors are small, so their detector noise-equivalent powers (NEPs) must be small to reach the background limit.

We present the first direct-detection submillimeter spectrometer, the South Pole Imaging Fabry-Perot Interferometer (SPIFI). SPIFI uses 25 silicon bolometers in the focal plane of an imaging system that matches each detector to the diffraction limit on the sky. To obtain high responsivity and low noise, the detectors are cooled to $60 \mathrm{mK}$ with an adiabatic demagnetization refrigerator (ADR). Two cryogenic scanning metal mesh Fabry-Perot interferometers (FPIs) provide spectral resolving power $R \sim \lambda / \Delta \lambda$ between 500 and 10,000 across the entire array, and careful filtration ensures good spectral purity in the system. SPIFI has been four years in the design, construction, and testing and has recently undergone successful commissioning runs at the JCMT. The instrument is unique: It can provide a wealth of useful astronomical observations in the coming years and serves as an example for future instrumentation development. In Section 2 we describe the instrument concept and technical details. In Section 3 we discuss the operation of SPIFI on the telescope, and in Section 4 we present the instrument's measured sensitivity along with a comparison with other spectrometers.

\section{Instrument Design}

\section{A. Overview}

In background-limited direct-detection spectroscopy, maximum sensitivity is obtained with the instrumental linewidth matched to the instrinsic width of the astronomical line. SPIFI is designed to observe a wide range of galactic and extragalactic targets; thus the instrument's resolving power $(R=\lambda / \Delta \lambda)$ should be variable, from $c / R=30 \mathrm{~km} \mathrm{~s}^{-1}$ for our Galactic Center, to $100-200 \mathrm{~km} \mathrm{~s}^{-1}$ for nearby galaxies, to as low as $500 \mathrm{~km} \mathrm{~s}^{-1}$ for distant galaxies. The highresolving-power end of this range requires a long interference path; $R$ of 10,000 at $370 \mu \mathrm{m}$ requires a (cryogenic) path length of approximately $\lambda R / 2=1.8$ $\mathrm{m}$, so a grating spectrometer would require a huge cryogenic volume. A FPI is a multipass spectrometer, and a $R=10,000$ etalon need only be a few centimeters thick, providing a substantial benefit in a cryogenic environment. FPI systems are also fully tunable; the resolving power is continuously variable by a change in the cavity spacing.

Spectroscopy with a FPI lends itself naturally to a spatial imaging system, allowing multiple detectors to be configured in a two-dimensional array on the sky. The spatial array is a substantial advantage for extended sources such as the Galactic Center and nearby starburst galaxies, providing increased speed and improved registration and calibration in the maps. Observations of unresolved sources, such as the ultraluminous infrared galaxies and highredshift galaxies, can also benefit from a spatial array; the source can be chopped between two pixels on the array, improving the sensitivity by $\sqrt{2}$. Furthermore, off-source pixels can be used to subtract much of the sky noise that is due to fluctuating telluric transmission inherent in ground-based submillimeter observations.

\section{B. Optical Design}

The instrument's optical design is based on a previously existing focal-plane assembly originally developed as an $850-\mu \mathrm{m}$ photometer by Swain et al. ${ }^{15}$ The 25 detectors are mounted in a square format, separated by $6.8 \mathrm{~mm}$. The spectrometer is designed to match this detector spacing to a $1.5 \lambda / D$ beam on the sky at $370 \mu \mathrm{m}$ and provide resolving power selectable from 500 to 10,000 over the full array.

As with any direct-detection spectrometer, a resolving power of 10,000 requires a collimated beam through the primary dispersing element because the maximum resolving power $R_{\max }$ in a converging (or diverging) beam is $R_{\max } \sim 2 f^{2}$, where $f$ is the focal 


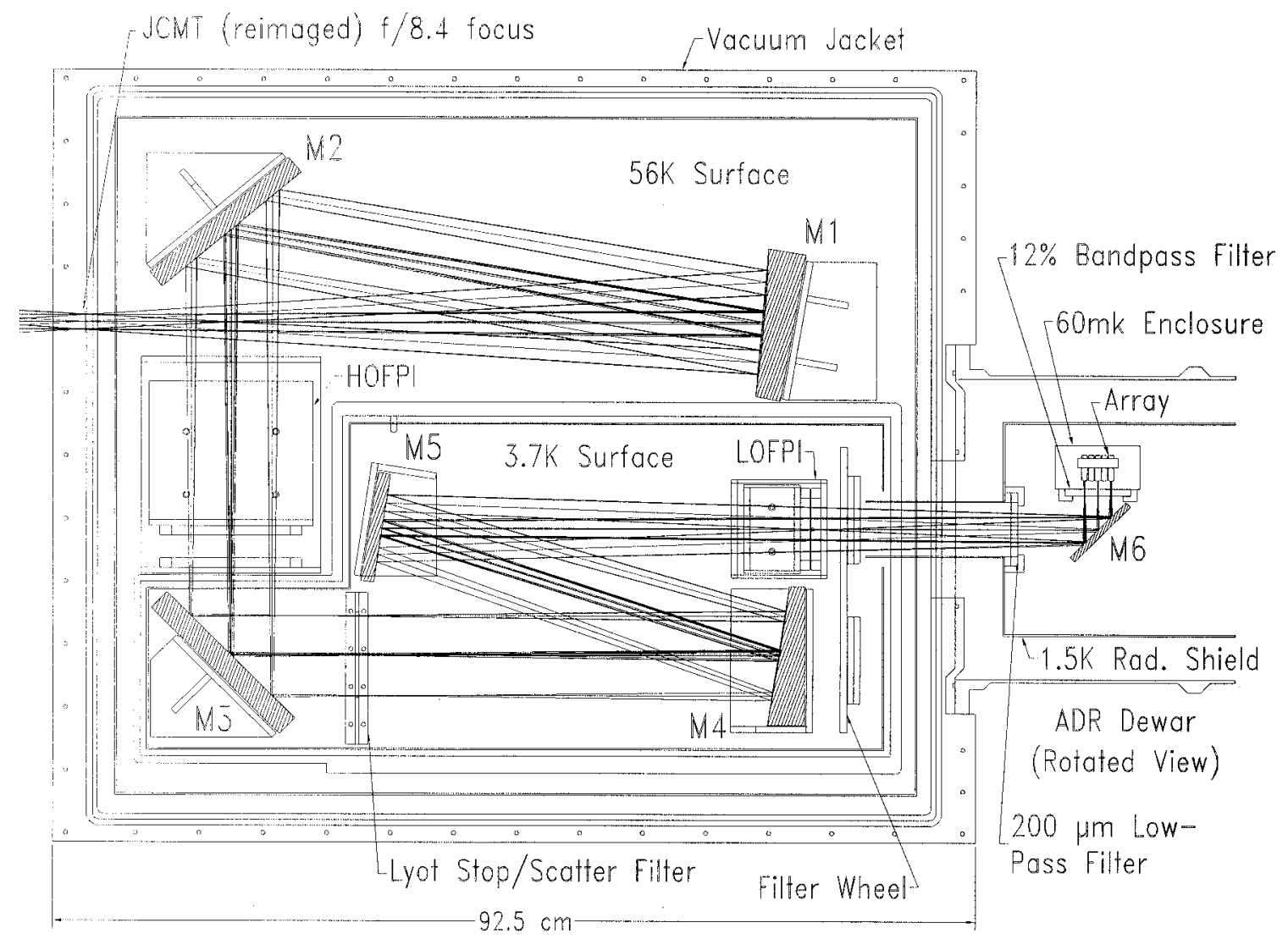

Fig. 1. SPIFI optical layout. The beam is near its $f / 8.4$ focus as it enters the cryostat through the polyethylene window. M1 is an off-axis paraboloid and collimates the beam at $9 \mathrm{~cm}$; M2 is a flat mirror that directs the collimated beam through the high-order FPI (HOFPI) and into the 3.7-K enclosure. Flat mirror M3 directs the beam through the cold aperture stop (Lyot stop) and scatter filter to the camera mirror M4, another off-axis paraboloid. The field is imaged at f/12.6 on the detector array by flat mirrors M5 and M6 after the beam transits the low-order FPI (LOFPI) and mesh filters at 1.5 and $0.06 \mathrm{~K}$. For clarity, M6 and the detector array are shown rotated $90^{\circ}$ about the beam axis as it is incident on M6 (see also Fig. 2).

ratio. Even with a collimated beam in the spectrometer, the finite solid angle subtended by a diffractionlimited sky beam limits the spectral resolution. As the incident beam is tipped relative to the etalon normal, a Fabry-Perot resonates at a shorter wavelength according to $\lambda=\lambda_{0} \cos \theta$. For small angles, this shift is second order in the off-axis angle $(\delta \lambda \sim$ $\theta^{2}$ ), so the spectral profile is both shifted and broadened for off-axis fields. The angular extent of a diffraction-limited field in the spectrometer's collimated beam scales inversely with the collimated beam size, so the degradation of resolution can be made negligible for a sufficiently large collimated beam inside the instrument. It can be shown that, to prevent resolution degradation from this effect, the size $D_{C}$ of the collimated beam must be at least ${ }^{16,17}$

$$
D_{C}=\left(2.25 \lambda^{2} R n_{\text {beams }}\right)^{1 / 2}
$$

where $R$ is the resolving power and $n_{\text {beams }}$ is the number of diffraction-limited beams from the center of the array to the corner. For SPIFI, we require $R=10,000$, and with our $5 \times 5$ array, $n_{\text {beams }}=2 \sqrt{2}$; thus the collimated beam inside the instrument must be $9 \mathrm{~cm}$ in diameter.

The size of the spectrometer and the optical layout presented in Fig. 1 are driven by this constraint. The input beam is $f / 8.4$ and reaches a focus near the Dewar window. The first mirror collimates the input beam at $9 \mathrm{~cm}$, and the second flat mirror sends the beam through the high-order FPI (HOFPI). Immediately after the HOFPI, the beam enters the ${ }^{4} \mathrm{He}$ temperature enclosure and is directed through the Lyot stop by flat mirror 3. Mirror 4 reimages the field at $f / 12.6$ for the detector array, which is reached after flat mirror 5, the low-order FPI (LOFPI), flat mirror 6 , and the $60-\mathrm{mK}$ bandpass filter. The radiation is coupled to the 1-mm-diameter detectors with compound parabolic cones, or Winston cones, ${ }^{18}$ each with an entrance aperture of $5.6 \mathrm{~mm}$. Because the cones are designed for an $f / 4$ beam, their response patterns are substantially oversized relative to the beam from the spectrometer, and their only effect on the optical system is to increase the effective area of the detectors.

All the mirrors are diamond-turned aluminum with a scratch-safe chromium-gold coating. The two powered mirrors are off-axis paraboloids and 


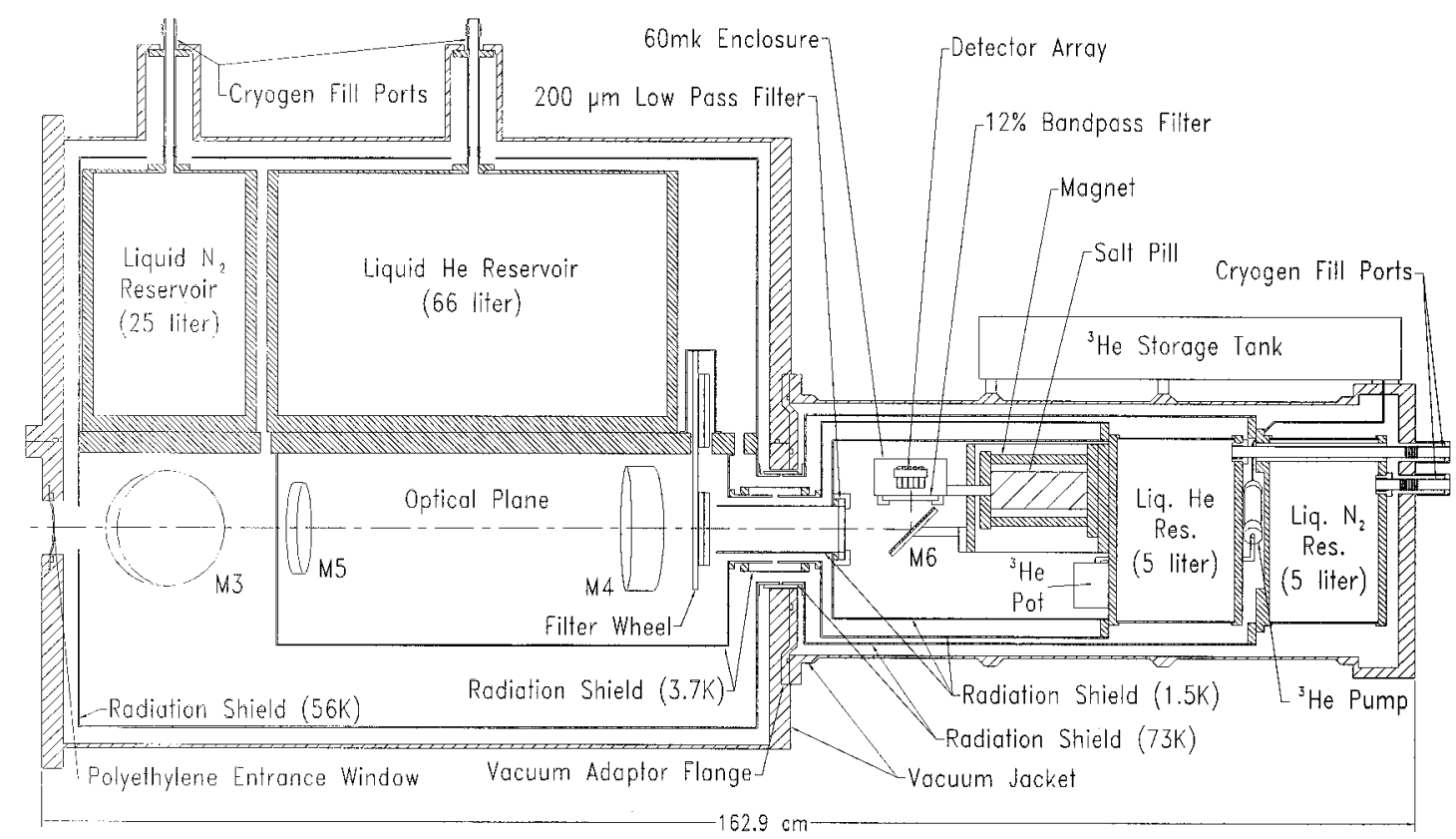

Fig. 2. SPIFI cryostat. Two independent cryostats joined together share a common vacuum and radiation shields. In the spectrometer cryostat, the liquid-nitrogen and helium-cooled optical benches lie below the cryogen vessels. A lengthwise split in this large vacuum vessel provides easy access to the optical components when it is inverted. The smaller detector cryostat supports the millidegrees Kelvin refrigerator (see Fig. 7), with liquid-nitrogen and pumped liquid-helium baths. The system of matching cylindrical snouts and closefitting connecting shields excludes ambient radiation to the level of $1 \%$ of the power in the spectrometer beam $\left(\sim 10^{-14} \mathrm{~W} /\right.$ detector in the $12 \%$ band).

are in a Czerny-Turner configuration ${ }^{19}$ to minimize coma. All optical elements except for the cold aperture stop are oversized by $30 \%$ to minimize diffraction effects from edges within the cryogenic instrument. Because there are only 25 detector elements in the focal plane, simple parabolic shapes are sufficient to ensure suitable optical performance. The geometric-ray spot sizes are less than $1 \mathrm{~mm}$ in the focal plane, completely negligible compared with the 5-mm diffraction spot.

\section{Cryogenic Design}

SPIFI combines two cryostats that can be operated independently; see Fig. 2. The Fabry-Perots and spectrometer optics are housed in a large cylindrical Dewar that is split along its length to provide easy access to the optical elements when the system is warm. The spectrometer optics and radiation shields are cooled with liquid nitrogen and helium cryostats constructed of welded aluminum and bolted above the aluminum optical benches. The $100 \mathrm{~kg}$ of cryogenic mass is suspended from the vacuum shell with four $1.6 \mathrm{~mm}$ thick $\times 50 \mathrm{~mm}$ wide $\times 50 \mathrm{~mm}$ long G-10 fiber-glass struts that allow the cryogenic assembly to remain centered with respect to the shell when cooled. To minimize the frequency of helium transfers, the helium cryostat occupies the bulk of the volume above the optical benches. Its 66-l capacity provides a helium hold time of five days; and with the ambient Mauna Kea atmospheric pressure, the bath operates with $T=3.7 \mathrm{~K}$. The nitrogen cryostat holds $22 \mathrm{l}$, and the vapor over the bath is pumped to
10 Torr to decrease the bath temperature to $56 \mathrm{~K}$. Cooling the optics and surfaces in the nitrogen stage lowers the background power on the detectors by $20 \%$, reducing its contribution to the photon noise by $10-20 \%$, depending on the operating conditions.

The detector array is housed in a smaller cryostat that supports the ADR with liquid nitrogen, ${ }^{4} \mathrm{He}$, and ${ }^{3} \mathrm{He}$ systems. The temperature of the walls of the ${ }^{4} \mathrm{He}$-cooled enclosure is crucial, because the resulting radiation field couples with the large solid angle $\left(\sim 100 \mathrm{deg}^{2}\right)$ and the large bandwidth $(\sim 1 / 8)$ to the entrance aperture of the detector feed cones. For this reason, and to ensure that no stray radiation leaks into the enclosure, there are two complete radiation shields mounted to the ${ }^{4} \mathrm{He}$ bath, and the bath is always pumped to 3 Torr, or $1.5 \mathrm{~K}$, so short submillimeter radiation from the surfaces is negligible. Aside from the ${ }^{4} \mathrm{He}$ consumed in recycling the millidegrees Kelvin systems, both cryostats have a hold time of more than $24 \mathrm{~h}$. The operation of the demagnetization and ${ }^{3} \mathrm{He}$ refrigerators is described in more detail in Subsection 3.F.

One of the unique features of SPIFI is the joining of the two independent cryostats in a manner that eliminates thermal IR radiation from the beam path. This is accomplished with pairs of matching cylindrical extensions, or snouts, that butt together, one each on the spectrometer and detector cryostats, for both the nitrogen and helium temperature radiation shields. Each pair of snouts is enclosed in a single radiation shield that overlaps and makes a close fit. Within these two connecting shields, a third long 


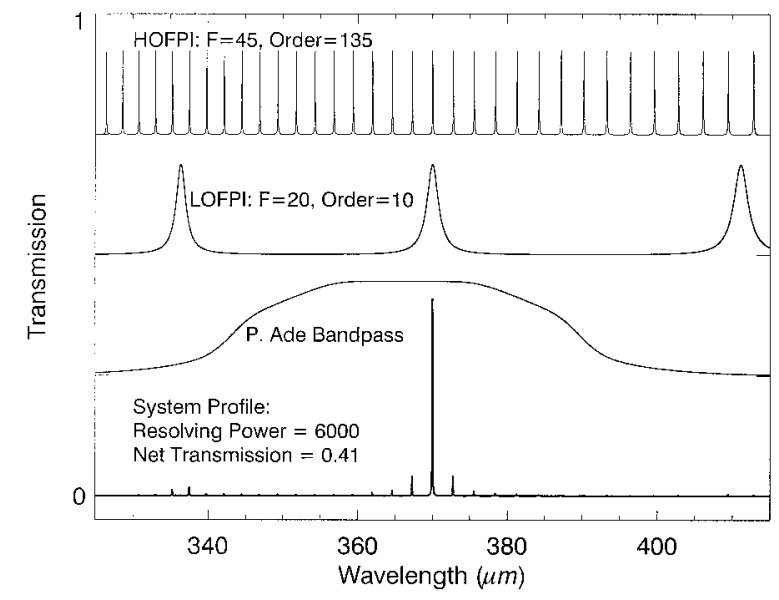

Fig. 3. SPIFI spectrometer with example configuration for $R=$ 6000. The spectral profiles of the three components in the spectrometer are plotted for an example setup (each offset and plotted at $1 / 4$ vertical scale). The net profile is the product, plotted with the thick curve at the bottom (full vertical scale). The peak transmission of 0.41 does not include losses in the window, mirrors, or the thermal IR blocking filters.

cylindrical extension protrudes from the detector cryostat's inner helium radiation shield to near the filter wheel in the spectrometer cryostat. With this arrangement, the stray radiation power on a detector with the instrument assembled is not measurably different from that of the closed $1.5-\mathrm{K}$ detector cryostat, to the level of $10^{-14} \mathrm{~W}$ on any given detector in our $12 \%$ band.

\section{Spectrometer}

\section{Triple Fabry-Perot Concept}

SPIFI obtains a resolving power $R$ up to 10,000 with three spectral elements: two cryogenic scanning FPIs and one fixed bandpass filter. The HOFPI provides the resolution of the instrument, the LOFPI, together with the fixed bandpass filter pass only, provides the desired order of the HOFPI, ensuring that there is little out-of-band power transmitted by the spectrometer and that the in-band transmission is fairly high. Figure 3 plots the spectral profiles of each of these elements and the resulting system spectral profile.

The resolving power of a Fabry-Perot etalon is the effective interference path length in units of wavelength equal to the product of the cavity order $m=$ $2 d / \lambda$ and with finesse $F$ approximately equal to the average number of reflections. ${ }^{20}$ The partially reflecting mirrors that form the cavity are characterized by a single-pass reflection coefficient $r$ that is typically greater than $90 \%$. This determines the cavity finesse according to $F=\pi \sqrt{r} /(1-r)$. The transmission of the etalon is determined by the absorption losses in the cavity mirrors, characterized by a single-pass absorption coefficient for a single mirror $a=1-t-r$. For $a<1.5 \%$, the cavity transmission scales approximately as $T_{\text {cavity }}=1-0.6 F a$. Typically, our mirrors have $a \sim 1 \%$, so it is important to keep the cavity finesse modest $(F \leq 60)$ to maintain high transmission.

In SPIFI, the HOFPI determines the instrumental bandwidth. It operates with finesse $F$ between 30 and 60 and order $m$ between 20 and 250 . Because the free spectral range of a FPI is $1 / m$, sorting two neighboring HOFPI orders from the desired order requires a resolving power $R_{\text {sort }} \sim m_{\text {HOFPI }}$. The broad wings of the LOFPI Lorentzian profile require that this resolving power be somewhat greater. As a minimum, $R_{\text {sort }}=1.5 \times m_{\text {HOFPI }}$ results in $20 \%$ power out of band. The LOFPI provides this resolving power with a finesse $F_{\text {LOFPI }}$ of 20-25 and the order $m_{\text {LOFPI }}$ between 5 and 15 . The LOFPI orders must then also be sorted; for this we employ a fixed interference filter. These bandpass filters are constructed for SPIFI by P. Ade. The filter shapes are more square than a Fabry-Perot Lorentzian profile, so that the FWHM of a $12 \%$ filter effectively sorts LOFPI orders up to 13 with less than $15 \%$ out-ofband leakage (see Fig. 3). Both cryogenic FabryPerots can be tuned arbitrarily, allowing observation of any frequency passed by the bandpass filter. At present, the filter cannot be changed in operation; we employ a filter centered at $366 \mu \mathrm{m}$, which is appropriate for our primary observing program of the $370-\mu \mathrm{m} \mathrm{CO}$ and $[\mathrm{CI}]$ lines. The second generation of the instrument under construction will allow a selection of three to four filters while cold.

\section{Implementation of the Fabry-Perot Interferometers}

Both of SPIFI's cryogenic FPIs claim heritage from the UC Berkeley-Max-Planck-Institute für extraterrestrische Physik Far-Infrared Fabry-Perot Interferometer (FIFI) ${ }^{17}$ and the Cornell Kuiper Widefield Infrared Camera (KWIC), ${ }^{21}$ far-IR spectrometers used on the Kuiper Airborne Observatory. The etalon mirrors are commercially available electroformed nickel mesh (Buckbee-Mears Corporation, St. Paul, Minn.), which is stretched and glued onto stainlesssteel rings. For research at $370 \mu \mathrm{m}$, we use mesh with a grid spacing of 85 and $64 \mu \mathrm{m}$, equivalent to 300 and 400 lines/in. The grid pattern is square, and the grid constant and area filling factor accurately predict the single-pass reflectivity $R$ and thus cavity finesse $F$ according to standard mesh theory. ${ }^{22}$ Unfortunately, although the grid constant of the mesh is well controlled, the area filling factor cannot be specified and varies from batch to batch. In practice, this prevents an arbitrary choice of finesse.

In both scanning FPIs, each of the two mesh rings is held magnetically to an aluminum frame. One of these frames is mounted rigidly to the optical bench, the other translates and so changes the cavity spacing. The two frames are joined in a parallelogram flex-vane construction (see Figs. 4 and 5). When viewed from the side, the moving and fixed frames form the top and bottom of a parallelogram. At either side are spring steel plates buttressed such that they bend only over small regions near their connection to the frames. This ensures that, for relatively 


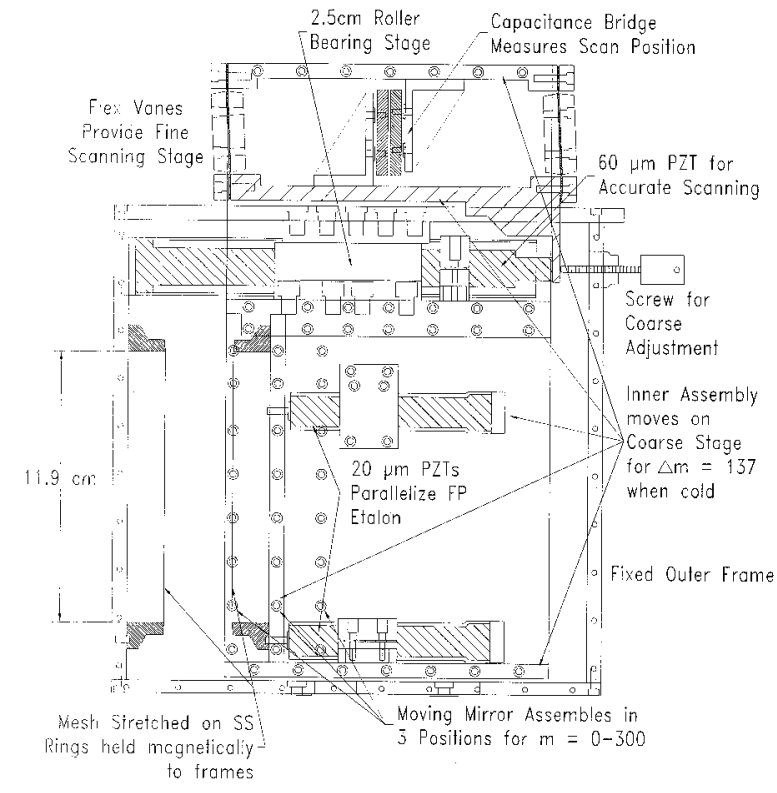

Fig. 4. SPIFI HOFPI with the outer sidewalls removed. The FPI mirrors are nickel mesh stretched and glued onto stainlesssteel (ss) rings (shown in section) held magnetically to the fixed and moving frames. To adjust the cavity order $m$ (here based on $\lambda=$ $370 \mu \mathrm{m}$ ), the entire inner assembly is translated on the rollerbearing stage by the adjustment screw. For spectral scanning, the PZT pushes the inner frame to the left against the bottom of the flex-vane parallelogram. The top of the flex-vane parallelogram is part of the inner frame; the bottom remains fixed to the outer frame with the adjustment screw. FP, Fabry-Perot.

small distances ( $\sim 5 \%$ of the Fabry-Perot size), the moving frame remains parallel to the fixed frame and the motion does not rely on any sliding, slipping, or rolling mechanism. The translation of this flexure system is the scanning of the Fabry-Perot etalon; it is actuated with a piezoelectric transducer (PZT) specially made for cryogenic use (Physik Instrumente Corporation, Waldbronn, Germany). The spacing of each of the HOFPI and LOFPI cavities is measured and controlled with a nulling capacitance bridge circuit that sets the PZT voltage. ${ }^{16}$ Plate spacing is controlled to within $0.3 \mu \mathrm{m}$ with a bandwidth of a few hertz.

Figure 4 shows the SPIFI HOFPI. It employs the largest cryogenic PZT available, with a cryogenic expansion range of $60 \mu \mathrm{m}$ at $60 \mathrm{~K}$. At $\lambda=370 \mu \mathrm{m}$, this is approximately a third of a free spectral range, or 12-20 times the spectral resolution element, depending on the cavity finesse. Hence the maximum scan length and the resolving power are inversely proportional; a higher-resolution setup allows smaller total bandwidth. In addition, the HOFPI incorporates a roller-bearing translation stage actuated with a fine adjustment screw that moves the entire inner assembly including the mirror, PZT, flex vanes, and capacitive sensor over a $2.5-\mathrm{cm}$ range. This provides a cryogenic adjustment range of 135 orders at $370 \mu \mathrm{m}$, or a change in resolving power $\Delta R$ from 4000 to 8000 , again depending on the finesse. Together with the roller-bearing stage, three assembly configurations

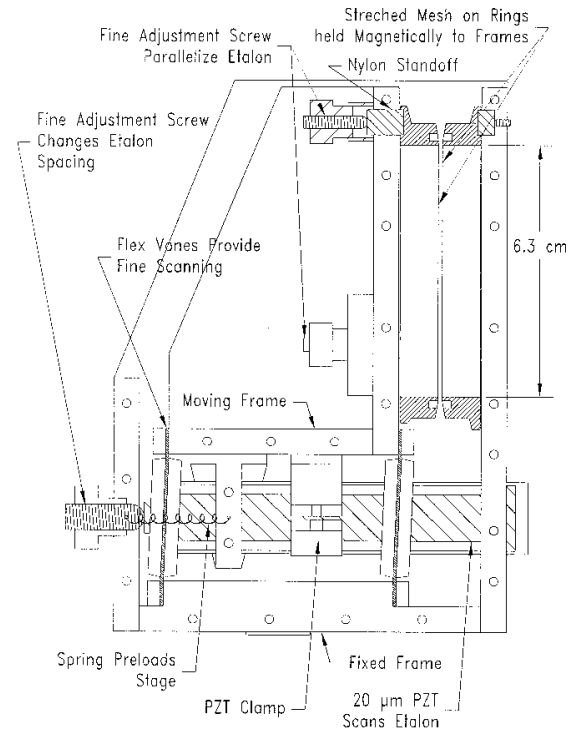

Fig. 5. SPIFI LOFPI with the sidewalls removed.

make available order $m$ up to 300 (for $\lambda=370 \mu \mathrm{m}$ ). The HOFPI mesh rings have a clear aperture of $12 \mathrm{~cm}$ to eliminate edge effects, and the diameter requires a thickness of $1.6 \mathrm{~cm}$ to carry an optically flat figure on which the mesh can be stretched. Once assembled and cold, the HOFPI etalon is parallelized with three $20-\mu \mathrm{m}$ PZTs and monitored with a He-Ne laser beam reflected from both meshes. This technique allows parallelism to better than $3 \times 10^{-5} \mathrm{rad}$, and the etalon is generally stable for several hours if the setup is not changed. Whenever coarse changes are made to the HOFPI spacing such as a change in the observing bandwidth or large changes in the frequency, the parallelism must be checked and the voltages of the parallelizing PZTs adjusted.

The laser retroreflection scheme also provides a means of determining the linearity of the spectral scan. The limitation arises in the counting of He-Ne fringes: Because there are a few tens of optical fringes in one spectral scan, both the linearity and the total length of the spectral scan can be determined only to $2-3 \%$.

The LOFPI shown in Fig. 5 is somewhat smaller with a 5-cm clear aperture. A PZT with a $15-\mu \mathrm{m}$ range in series with a fine adjustment screw actuates the flexure stage through a total range of around 20 orders or $4 \mathrm{~mm}$. Because the cavity spacing is small, the meshes themselves are used as the capacitive sensor in the LOFPI bridge. Because it is a simpler mechanical device, has smaller mirrors, and operates at lower resolving power, the parallelism of the LOFPI etalon does not require cryogenic adjustment. The mesh rings are adjusted warm, and when cold the etalon is parallel to better than $2 \times 10^{-4} \mathrm{rad}$, more than adequate for its resolving power of a few hundred.

\section{Filtration}

Bolometric detectors respond to all frequencies that can couple power to the absorber, so careful attention 
Table 1. Elements in SPIFI Optical Train

\begin{tabular}{lccc}
\hline \multicolumn{1}{c}{ Element } & $T(\mathrm{~K})$ & Spectral Property & $\begin{array}{c}\text { Transmission } \\
\text { at } 370 \mu \mathrm{m}\end{array}$ \\
\hline $\begin{array}{l}\text { Polyethylene } \\
\text { entrance window }\end{array}$ & 280 & & 0.96 \\
Mirrors (six) & 280 & & \\
HOFPI & 56 & $\frac{\lambda}{\Delta \lambda} \sim 500-10,000$ & $0.98)^{6}$ \\
Lyot stop scatter & 3.7 & $\lambda>100 \mu \mathrm{m}$ & 0.9 \\
$\quad$ filter & 3.7 & $\frac{\lambda}{\Delta \lambda} \sim 80-250$ & 0.75 \\
$\begin{array}{l}\text { LOFPI } \\
\text { Low-pass mesh filter }\end{array}$ & 1.5 & $\frac{\lambda}{\lambda}>200 \mu \mathrm{m}$ & 0.85 \\
Bandpass filter & 0.06 & $\frac{\lambda}{\Delta \lambda}=8.8$ & 0.78 \\
Total & & $\frac{\lambda}{\Delta \lambda} \sim 500-10,000$ & 0.26 \\
\hline
\end{tabular}

must be paid to filtration of stray radiation, particularly in a low-background instrument such as SPIFI. Blocking short wavelength radiation ( $\lambda$ between 5 and $100 \mu \mathrm{m}$ ) is especially critical because, at these wavelengths, the FPIs have small but nonzero transmission and the bandpass filters invariably have leaks. A small leak in the filter train at short wavelengths can result in substantial power on the detector because radiation sources warmer than $50 \mathrm{~K}$ emit much more power at the shorter wavelengths than in the submillimeter wavelengths. In addition to the Fabry-Perots and bandpass filter, SPIFI employs two short wavelength blocking filters constructed by P. Ade to eliminate stray thermal IR power. The first, at the Lyot stop, is a flexible scatter filter on a polyethylene substrate. The second is a reflective mesh filter, positioned at the entrance to the $1.5-\mathrm{K}$ stage of the ADR cryostat. Table 1 lists the elements in SPIFI's optical and filter train, along with its transmission in our $370-\mu \mathrm{m}$ band. Tabulating each of these values, we calculate a net transmission from the front of the instrument to the detectors of approximately 0.25 , a value consistent with measurements of the quantum efficiency transmission product.

\section{E. Bolometric Detectors}

SPIFI's detectors are silicon bolometers provided by S. H. Moseley and C. A. Allen at NASA Goddard Space Flight Center, Greenbelt, Maryland. They are similar to the bolometers used in the Submillimeter High Angular Resolution Camera (SHARC) ${ }^{14}$ and microcalorimeters used for x-ray spectroscopy. ${ }^{23,24}$ Operational parameters of SPIFI's detectors are presented in Table 2 . The range in values reflects both the variation in operating conditions as the spectral resolution is changed and some variation across the array. Each detector is etched from a single piece of silicon and consists of a frame, four support legs, and the detector itself, a $1 \mathrm{~mm}$ diameter $\times 12 \mu \mathrm{m}$ thick disk. The support legs provide the thermal conductance from the detector to the
Table 2. SPIFI Detector Parameters

\begin{tabular}{lcc}
\hline \multicolumn{1}{c}{ Parameter } & Value & Unit \\
\hline Bath temperature & 60 & $\mathrm{mK}$ \\
Thermal conductance $G$ & $2-3 \times 10^{-11}$ & $\mathrm{~W} / \mathrm{K}$ \\
Operating temperature & $80-110$ & $\mathrm{mK}$ \\
Dark impedance & $5-100$ & $\mathrm{M} \Omega$ \\
Operating impedance & $0.1-5$ & $\mathrm{M} \Omega$ \\
Optical quantum efficiency $\eta$ & 0.5 & \\
Electrical responsivity $S_{e}{ }^{a}$ & $3-10 \times 10^{8}$ & $\mathrm{~V} / \mathrm{W}$ \\
Operating Johnson noise ${ }^{b}$ & $3-10$ & $\mathrm{nV} / \sqrt{\mathrm{Hz}}$ \\
Operating thermal noise & $3-7$ & $\mathrm{nV} / \sqrt{\mathrm{Hz}}$ \\
Thermistor 1/f noise at $8 \mathrm{~Hz}$ & $15-60$ & $\mathrm{nV} / \sqrt{\mathrm{Hz}}$ \\
Amplifier noise & 5 & $\mathrm{nV} / \sqrt{\mathrm{Hz}}$ \\
Total nonphoton contribution to NEV & $15-60$ & $\mathrm{nV} / \sqrt{\mathrm{Hz}}$ \\
Total photon noise contribution to NEV & $35-40$ & $\mathrm{nV} / \sqrt{\mathrm{Hz}}$ \\
\hline${ }^{a}$ Electrical responsivity does not include the optical $\mathrm{quantum}$ \\
efficiency. \\
${ }^{b}$ All noise-equivalent voltages (NEVs) refer to the detector.
\end{tabular}

frame, and two of the legs are degenerate boron doped to provide an electrical connection to the thermistor. The thermistor is a small $\left(\sim 0.1 \mathrm{~mm}^{2} \times 0.5 \mu \mathrm{m}\right.$ thick $)$ phosphorus-doped region with $50 \%$ boron compensation implanted on the circular detector. The conduction in the thermistor is a hopping mode within the valence band; thus each device has a temperaturedependent resistance given by $R=R_{0} \exp \left(\sqrt{T_{0} / T}\right)$, with $R_{0}=60-70 \Omega$, and a range of $T_{0}$ from 7 to $12 \mathrm{~K}$. The impedance of the dark detectors at $60 \mathrm{mK}$ varies across the array from 5 to $100 \mathrm{M} \Omega$, but this drops to 0.4-10 $\mathrm{M} \Omega$ as the detectors are illuminated by the ambient background power $(0.5-1.5 \mathrm{pW})$. The thermal conductance through the legs to the silicon frame is $G=2-3 \times 10^{-11} \mathrm{~W} / \mathrm{K}$, and thus the detectors are operating at around $\Delta T=P / G=75-90 \mathrm{mK}(15-30$ $\mathrm{mK}$ above the $60-\mathrm{mK}$ bath) when exposed to the light of the spectrometer. To increase the submillimeter absorption, a $120-\AA$ layer of bismuth is applied to the detector surface, matching the surface impedance to that of free space. For a typical detector, our measurements indicate a quantum efficiency of 0.5 , in agreement with the theoretical prediction for a metallic film on a dielectric substrate. ${ }^{25}$ Each detector is mounted in an Invar structure that forms the closed end of a 2-mm-diameter integrating cavity. This Invar cap is mounted to the base of the goldcoated copper Winston cone that couples the radiation from the focal plane into the integrating cavity.

The bolometers are each biased in series, as shown in Fig. 6, with a metal film chip resistor with a temperature-independent resistance of $29 \mathrm{M} \Omega$, mounted directly to the back of the Invar cap. Thus the bolometers are to first order current biased with a typical bias voltage of $20 \mathrm{mV}$ across the load resistor and the bolometer producing a detector current of a few $10^{-10} \mathrm{~A}$. Table 2 includes the power-to-voltage responsivity of the detectors, as well as the two fundamental noise sources, Johnson noise and thermal noise, that are estimated in the standard manner. ${ }^{25,26}$ The magnitudes of these noise sources depend on the radiant power incident on each detector that is a 


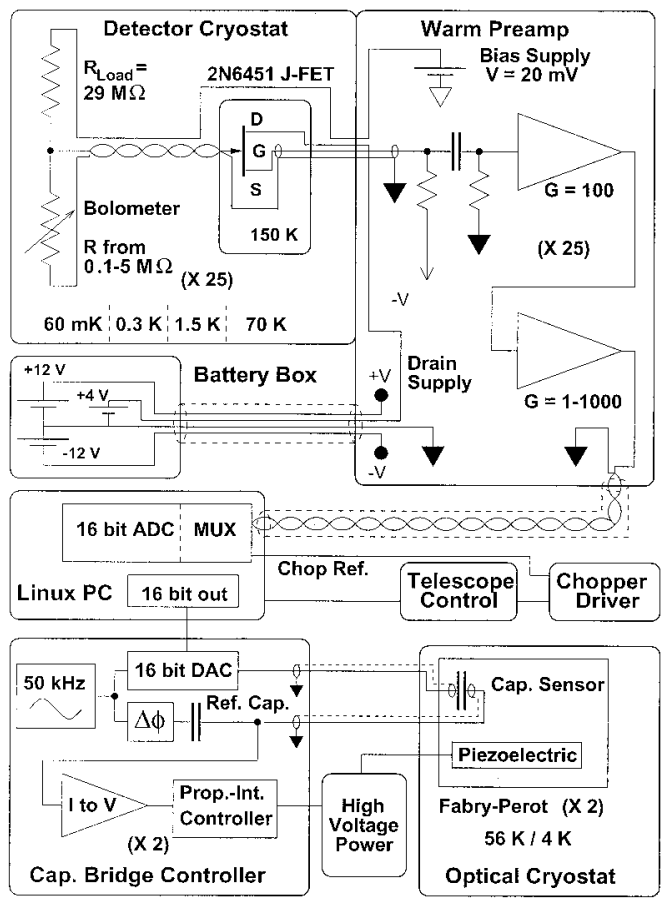

Fig. 6. SPIFI electronics block diagram. Each of the bolometers is biased in series with a $29-\mathrm{M} \Omega$ load resistor and buffered with a cooled junction FET (J-FET). To minimize pickup, all signal wires are twisted pairs or coaxial, the FETs and amplifiers are powered with batteries, and the preamplifier box is bolted tight to the cryostat. Also shown are the essentials of the capacitance bridge circuit and its connection to the FPIs. Dashed lines indicate shielded cables. ADC, analog-to-digital converter; DAC, digital-to-analog converter; MUX, multiplexer.

function of the resolving power, as well as on the fundamental detector properties that vary somewhat across the array. In addition to the Johnson and thermal noise, the doped silicon thermistors have additional $1 / f$ resistance noise that is intrinsic to the conduction in the bulk thermistor, i.e., not due to leads or contacts. ${ }^{27}$ Han et al. (1998) ${ }^{27}$ have characterized the dependence of the noise on the thermistor volume, temperature parameter $T_{0}$, and operating temperature $T$. The dependence is strong, and because it is a resistance noise, the measured voltage noise also scales with the operating bolometer voltage. Furthermore, the noise depends sharply on the thermistor doping levels that vary across SPIFI's array. This $1 / f$ noise contributes between 15 and 60 $\mathrm{nV} / \sqrt{\mathrm{Hz}}$ and is typically the dominant nonphoton noise contribution in the system.

\section{F. Millidegrees Kelvin Cryogenics}

SPIFI's bolometer array is cooled by adiabatic demagnetization of a paramagnetic salt. The method utilizes the fact that the thermodynamics of salt below approximately $1 \mathrm{~K}$ depend only on the ratio of the applied magnetic field $B$ to the temperature $T$; if properly thermally isolated, a decrease in $B$ results in a decrease in $T$ by the same ratio. The technique has been used for decades to cool samples in the

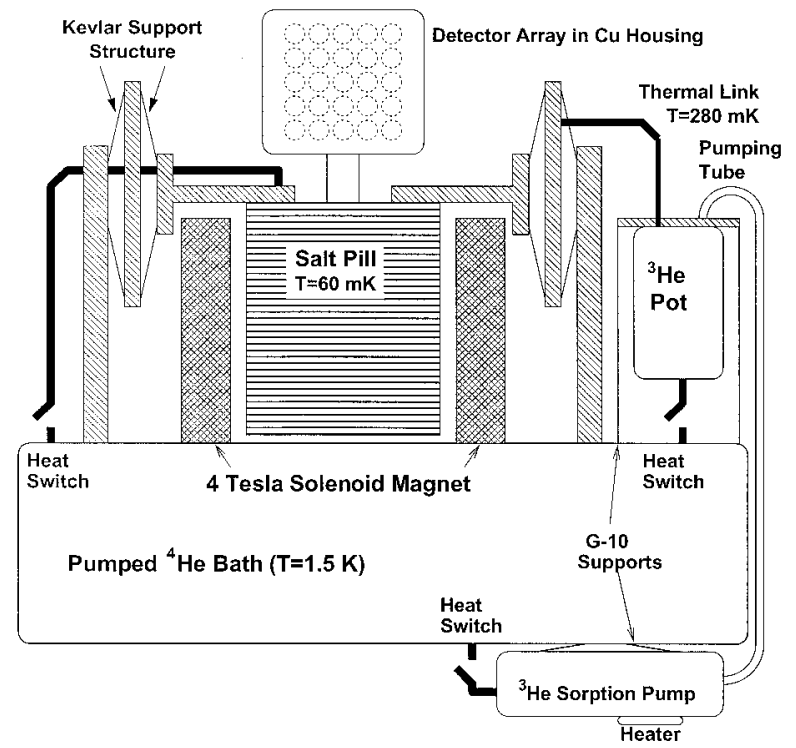

Fig. 7. ADR schematic. A network of Kevlar threads support the 60-mK stage and its 300-mK thermal guard. Mechanical heat switches connecting the salt pill and ${ }^{3} \mathrm{He}$ pot to the $1.5-\mathrm{K}$ bath are closed when the systems are recycled. The ${ }^{3} \mathrm{He}$ pump heat switch is opened and the pump is heated for the recycling, forcing ${ }^{3} \mathrm{He}$ to condense in the pot. In operation, the heat switches are reversed. The hold time of the system is around $40 \mathrm{~h}$, limited by conduction through the fiber-glass mounting of the ${ }^{3} \mathrm{He}$ pot.

laboratory 28,29 and recently for astronomical x-ray spectroscopy from sounding rockets. ${ }^{30}$ We outline our implementation here.

The salt used in SPIFI is $\mathrm{FeNH}_{4}\left(\mathrm{SO}_{4}\right) \cdot 12 \mathrm{H}_{2} \mathrm{O}$, called ferric ammonium alum. Demagnetization of this material from a starting temperature of a pumped He bath (1.5-1.8 K) can generate temperatures as low as $50 \mathrm{mK}$. Our salt pill was constructed by M. Dragovan at Yerkes Observatory; it is formed into a cylindrical pill of approximately $230 \mathrm{~g}$ and threaded with gold wires that promote good thermal conduction between the pill and the copper detector assembly. To minimize its heat load, the entire cold stage is mounted with a network of Kevlar threads to a ${ }^{3} \mathrm{He}$ stage. Surrounding the pill but mounted to the ${ }^{4} \mathrm{He}$ temperature structure is a superconducting solenoid magnet (American Magnetics Corporation) that provides a central field of $4 \mathrm{~T}$ with $20 \mathrm{~A}$ of current.

The cooling cycle begins when we magnetize the salt while it is thermally connected to the ${ }^{4} \mathrm{He}$ stage through a copper clamp heat switch. The heat of magnetization increases the temperature of the salt to around $10 \mathrm{~K}$, and $3 \mathrm{~h}$ are required for it to return to equilibrium with the pumped He bath. Once it has cooled back to $1.5 \mathrm{~K}$, we isolate the pill (and detector housing) from the bath by opening the heat switch, and the magnetic field is slowly removed at a constant rate over a period of $50 \mathrm{~min}$. Within $30 \mathrm{~min}$ after the demagnetization is complete, the pill and detector housing equilibrate near $60 \mathrm{mK}$. Access to the superconducting magnet portion of the circuit is 
possible through a persistence switch, a portion of the superconducting circuit that is heated to become normal, providing a resistance in parallel with the magnet's inductance. A commercial automatic ramping power supply and controller are employed for repeatable cycling of the system.

A thermal schematic of the refrigerator is shown in Fig. 7. Because the demagnetization refrigerator alone is incapable of supporting the conductive loads from $1.5 \mathrm{~K}$ through the wiring and the mechanical mounting structure, a ${ }^{3} \mathrm{He}$ system is employed as a thermal guard. This is a closed-cycle system consisting of a condensing pot and charcoal pump, both with copper clamp heat switches to the ${ }^{4} \mathrm{He}$ bath. Cycling the ${ }^{3} \mathrm{He}$ system begins when we isolate the pump and heat it to around $50 \mathrm{~K}$ to force the gas to condense in the pot, which is thermally connected to the $1.5-\mathrm{K}$ bath through its heat switch. This process also requires $3 \mathrm{~h}$, both to force the ${ }^{3} \mathrm{He}$ out of the pump and to remove the heat of vaporization of the condensing gas in the pot. The pump is then connected to the $1.5-\mathrm{K}$ bath by its heat switch, and the pot is isolated so that the liquid ${ }^{3} \mathrm{He}$ can reach its equilibrium temperature of $280 \mathrm{mK}$. When the instrument is at room temperature, the ${ }^{3} \mathrm{He}$ resides in a built-in external storage tank $(\mathrm{V} \sim 2 l)$ under three atmospheres of pressure.

The hold time of the 60-mK cold stage is limited by the hold time of the ${ }^{3} \mathrm{He}$ guard. The integrated heat capacity of the salt pill for a temperature change of 10 $\mathrm{mK}$ is $23 \mathrm{~mJ}$, which easily supports its heat loads. Both of the dominant loads, conduction from $300 \mathrm{mK}$ through the array wiring and radiation from the 1.5-K environment, are around $20-30 \mathrm{nW}$, limiting the hold time to around $100 \mathrm{~h}$. For the ${ }^{3} \mathrm{He}$ stage, the dominant heat load is conduction from $1.5 \mathrm{~K}$ through its fiber-glass mounting structure. This power is $75 \mu \mathrm{W}$ and vaporizes the $0.26 \mathrm{~J}$ of liquid ${ }^{3} \mathrm{He}$ in approximately $40 \mathrm{~h}$. In preparation for Antarctic observations, the second-generation instrument will achieve a several-day hold time either with an increase in the amount of ${ }^{3} \mathrm{He}$ or with the ${ }^{3} \mathrm{He}$ pot remounted with a higher thermal-impedance structure.

\section{G. Electronics and Software}

A schematic of the bolometer amplification electronics is shown in Fig. 6. As is customary with bolometers, the first stage for each channel is a junction field-effect transistor (FET) in a source-follower configuration. In SPIFI the FETs are assembled on two boards with 16 FETs each, and the boards are suspended from the ADR nitrogen stage such that the FETs heat themselves to around $150 \mathrm{~K}$. The detectors are connected to the FETs by twisted pairs of 0.1-mm-diameter constantan wire woven into ribbon cable (Oxford Instruments). To minimize the conductive losses to the cold stages, the cable is thermally sunk to ${ }^{3} \mathrm{He}-,{ }^{4} \mathrm{He}-$, and $\mathrm{N}_{2}$-cooled surfaces and is tensioned throughout to minimize microphonic pickup. After the junction FET buffers, the signals enter into a warm preamplifier bolted directly to the
ADR cryostat. The signals are ac coupled with a $\tau=$ 1-s filter before a low-noise $G=100$ amplifier and a programmable gain amplifier. The net gain of the cryogenic and warm electronics is variable from $10^{2}$ to $10^{5}$, and the total voltage noise is $5 \mathrm{nV} / \sqrt{\mathrm{Hz}}$, referred to the detector at our chopping frequency of 8 $\mathrm{Hz}$. Because the junction FETs are cooled, their current noise is less than $6 \times 10^{-16} \mathrm{~A} / \sqrt{\mathrm{Hz}}$, which results in a negligible $3 \mathrm{nV} / \sqrt{\mathrm{Hz}}$ when drawn through even a high-impedance (5-M $\Omega$ ) bolometer.

To minimize electronic pickup, the bias voltage is provided by a battery inside the preamplifier enclosure. The other cryogenic and warm electronics are powered with rechargeable lead-acid batteries, housed in a shielded box connected to the preamplifier with a shielded cable. After the warm preamplifier, the signals are sent through shielded, twisted pair cables to two Keithley DAS-1800ST/HR 16channel, 16-bit data-acquisition boards inside a PC operating with Linux. The data are processed with a custom software package in the MATLAB environment. The software commands the Fabry-Perot positions through a spectral scan, and at each position coadds the data stream from each channel with the chop reference as a template.

\section{Operation at the James Clark Maxwell Telescope}

SPIFI mounts at the right-hand Nasmyth platform, approximately $4 \mathrm{~m}$ from the center of the JCMT telescope. The beam is delivered from the nominal $f / 12$ focus inside the receiver cabin to an $f / 8.4$ focus just in front of the instrument by two lenses mounted in the telescope elevation structure. The lenses are diamond machined from plate stock blanks of highdensity polyethylene with a refractive index of 1.52 in the submillimeter. ${ }^{31}$ Both lenses are plano convex, with diameters of 13 and $16 \mathrm{~cm}$ and mean thicknesses of $6-7 \mathrm{~mm}$. The total transmission of the lens pair is $75 \%$.

Measurements of the sky brightness indicate that $65 \%$ of the power is coupled from the telescope focus to the sky within a few minutes of arc of the boresight, the remainder lost to scattering, spillover to the ground and dome, and ohmic losses on the dish. This defines the forward coupling $\eta_{F}$. On the basis of our mapping of this forward beam (Fig. 8), 21\% is in the main lobe, well reproduced as a FWHM of 7.8-arc min Gaussian; this is the main beam efficiency $\eta_{\mathrm{MB}}$. The total coupling from the main beam on the sky to the telescope focus is the product of these, $14 \%$. The measured size of the main lobe is somewhat larger than the nominal diffraction limit for the JCMT $(1.2 \lambda / D=6.1$ arc min), but both the size of the main lobe and its coupling intensity are consistent with the other measurements at the JCMT at these frequencies. ${ }^{32,33}$ The larger beam size means that the coupling to a true point source is even lower than the main beam efficiency; for this we define an additional quantity $\eta_{A}$, a measure of the frac- 


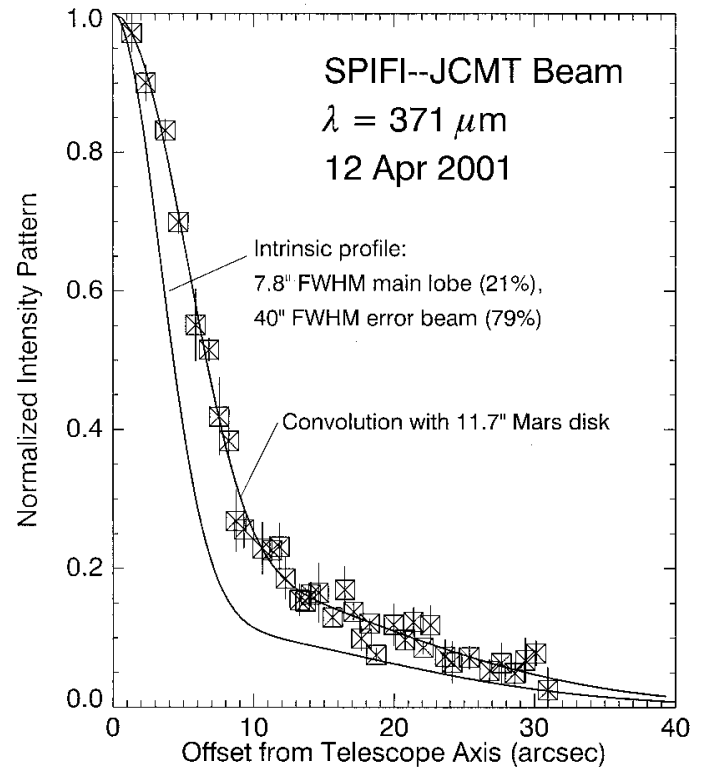

Fig. 8. SPIFI forward beam map at the JCMT. The observed pattern is the two-dimensional convolution of the Mars disk and the telescope beam pattern; at high frequencies, this is dependent on the conditions of the dish at the time of observation. This profile, measured in April 2001, is well fit with two Gaussian components, a 7.8-arc sec main lobe and a 60 -arc sec error lobe.

tion of the main beam that couples to a true point source:

$$
\eta_{A}=\frac{\lambda^{2}}{A_{\mathrm{tel}} \Omega_{\mathrm{MB}}} .
$$

Note that $\eta_{A}$ can be made unity if $A_{\text {tel }}$ is decreased; it is another way of expressing the effective aperture of the telescope. As defined above, our measurements at the JCMT give $\eta_{A}=0.48$. The total coupling from a point source to the telescope focus is then given by the product $\eta_{F} \eta_{\mathrm{MB}} \eta_{A}$.

To date, SPIFI has made five observing runs at the JCMT: in April and September 1999, May and September 2000, and May 2001. Observations in the short submillimeter windows are possible only in the driest conditions on Mauna Kea. Only the first and fifth runs have permitted useful observations at 370 $\mu \mathrm{m}$, as the conditions during the other runs were such that the zenith transmission was never above $10 \%$. During the April 1999 run, SPIFI had 12 functional pixels and was operating at $R=4800$; we were able to map the central $2 \mathrm{pc} \times 3 \mathrm{pc}$ of the galaxy in the $\mathrm{CO}$ $(J=7 \rightarrow 6)$ rotational transition with 15 pointings of the 12-pixel array (see Fig. 9). We also were able to obtain a single-array pointing of $\mathrm{CO}$ spectra toward the nucleus of the nearby starburst galaxy NGC 253 (see Fig. 10). These scientific results and their implications are in preparation and will be presented in separate articles.

Because the response of the bolometer array is a nonlinear function of the bandwidth, the instrument response must be calibrated whenever the observation setup is changed. To accommodate this, we employ a

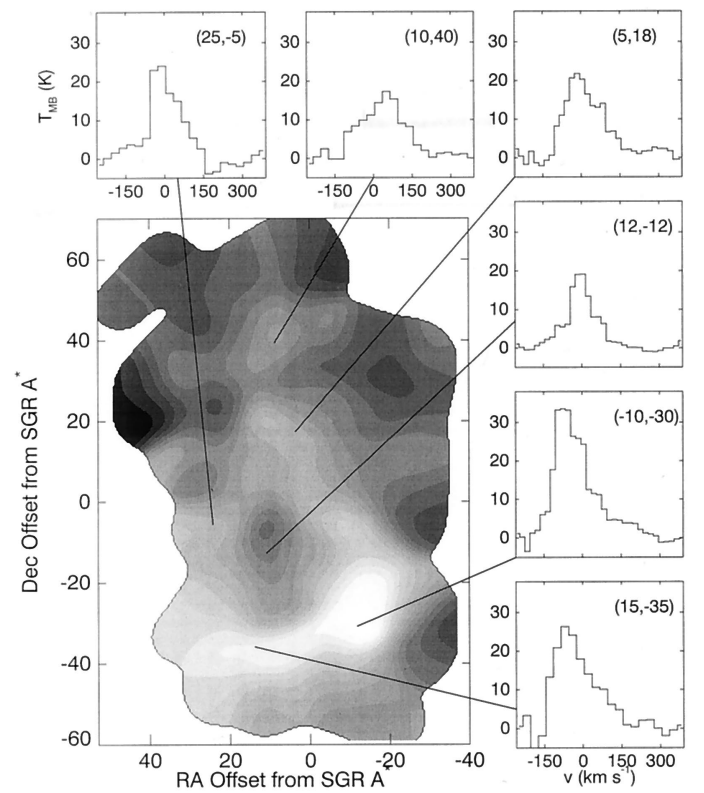

Fig. 9. Integrated intensity map of the $\mathrm{CO} J=7 \rightarrow 6(370-\mu \mathrm{m})$ emission from the Galactic Center circumnuclear disk (CND). Offsets are in seconds of arc relative to the dynamical center of the galaxy SGR $A^{*}\left(\mathrm{RA}_{1950}=17^{\mathrm{h}} 42^{\mathrm{m}} 29^{\mathrm{s}} .3, \operatorname{Dec}_{1950}=-28^{\circ} 59^{\prime} 19^{\prime}\right)$, and the beam size is 11-arc sec FWHM. Emission traces warm dense molecular gas and are observed throughout the map; contours are linear in $T_{\mathrm{MB}} \Delta \nu$, with a $250-\mathrm{K} \mathrm{km} \mathrm{s}^{-1}$ interval. The peak in the southwest CND is $5400 \mathrm{~K} \mathrm{~km} \mathrm{~s}^{-1}$; the minimum at the edges of the map is $750 \mathrm{~K} \mathrm{~km} \mathrm{~s}^{-1}$. Velocity resolution in the spectra is $50 \mathrm{~km} \mathrm{~s}^{-1}$. Overall rotational motion is evident in the shifting of the velocity from north to south. RA, right ascension; Dec, declination.

calibration unit permanently mounted to the front of the instrument for both flux and spectral calibration. A flip mirror sends the spectrometer beam to a collimating mirror and then a flat mirror, so that an image of the instrument pupil is formed outside the cryostat. At this exterior pupil is a piece of glass matched to the beam size heated at $40 \mathrm{~K}$ above the ambient temperature. A rotary blade chops in front of this heated glass, providing a flux calibration load that is identical for any position in the focal plane. In addition, a second rotary blade that chops immediately in front of the cryostat entrance allows chopping the ambient blade against the sky or alignment loads. Absolute flux calibration of astronomical sources is always based on planets, as they primarily couple to the main beam and not the large JCMT error beam.

For spectral calibration of SPIFI, we use $\mathrm{CO}$ or $\mathrm{H}_{2} \mathrm{~S}$ gas in a cell transited by the beam of our exterior calibration unit. The beam originating at the glass load transits the $50-\mathrm{cm}$ gas cell twice before entering the Dewar by way of the flip mirror, for a total path length of approximately $1 \mathrm{~m}$. With this arrangement, a cell pressure of a few torr provides sufficient column density for a high signal-to-noise ratio (SNR) absorption line spectrum in either $\mathrm{CO}$ or $\mathrm{H}_{2} \mathrm{~S}$. Observation of two lines in a single scan determines the range of the spectral scan, with a precision that depends on the SNR of the absorption features, but is 


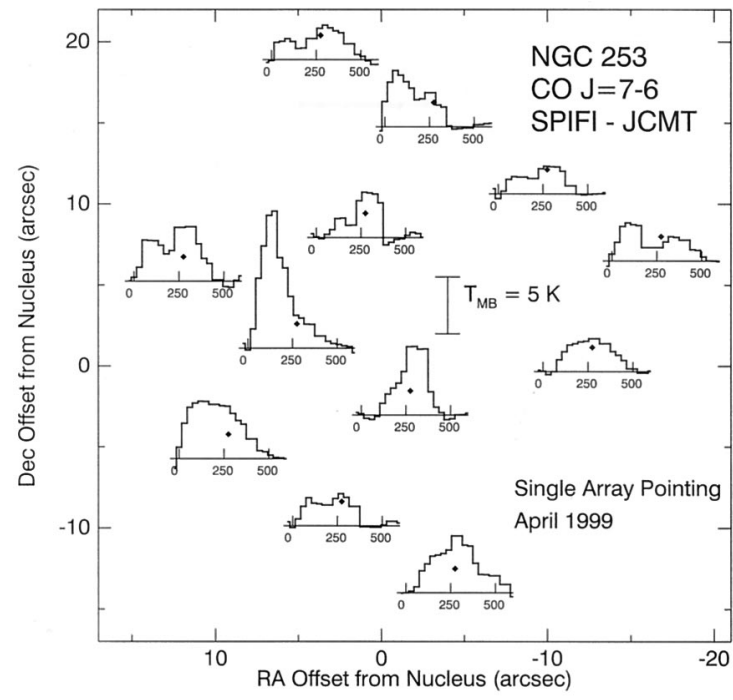

Fig. 10. $\mathrm{CO}(J=7 \rightarrow 6)$ in the starburst galaxy NGC 253. These data are a single footprint of the array from our first run at the JCMT in April 1999 and were obtained in 15 min of on-source integration time with atmospheric transmission to the source between 5 and $8 \%$. The data indicate widespread excited molecular gas in the nuclear starburst of NGC 253. RA, right ascension; Dec, declination.

typically better than $2 \%$. The scan linearity is described in Subsection 2.D.2.

Submillimeter sky transmission on Mauna Kea is monitored with radiometers operating near $200 \mathrm{GHz}$, at both the JCMT and the Caltech Submillimeter Observatory. The measured $200-\mathrm{GHz}$ opacities can be converted to a precipitable water-vapor burden, the depth of the resulting liquid layer if all the atmospheric water vapor were condensed. ${ }^{34}$ The optical depth at our observing frequency is then calculated according to the most recent Fourier-transform spectroscopy observations and modeling of Pardo, Cernicharo, and Serabyn. ${ }^{34,35}$ These analyses incorporate the opacity of dry air and provide a relationship between the precipitable water-vapor burden and the opacity at the submillimeter wavelengths. As an example, for our primary observing frequency of 810 $\mathrm{GHz}$, the opacity is given by $\tau_{810}=0.84+1.49 \times$ the precipitable water-vapor burden. We verified this relationship at the JCMT by measuring the emission of the sky as a function of zenith angle and fitting the result to a uniform-temperature atmosphere. With this relationship, sky dips are not needed as long as the $200-\mathrm{GHz}$ radiometers are in operation.

\section{Sensitivity}

The natural measure of sensitivity in a direct-detection system is the NEP (watt $/ \sqrt{\mathrm{Hz}}$ ) or noise-equivalent flux density (NEFD) $(\mathrm{Jyr} / \sqrt{\mathrm{Hz}})\left(1 \mathrm{Jyr}=10^{-30} \mathrm{~W} \mathrm{~cm}^{-2}\right.$ $\mathrm{Hz}^{-1}$ ). These quantities are defined in terms of the SNR, easily measured with a calibration load or astronomical source. The relationship is given by

$$
\mathrm{SNR}=\frac{P_{\mathrm{obs}} \sqrt{2 t_{\mathrm{int}}}}{\mathrm{NEP}}
$$

where $P_{\mathrm{obs}}$ is the signal power, $t_{\mathrm{int}}$ is the integration time, and the $\sqrt{2}$ is according to the convention for expressing the NEP with units of $\mathrm{W} / \sqrt{\mathrm{Hz}}$. The NEP is a general quantity that can refer to power on the detector, at the front of the instrument, or in the main beam on the sky. The NEFD is generally referred to a point source above the atmosphere. There are few point sources with sufficient flux for an $800-\mathrm{GHz}$ spectrometer, but planets tend to fill the main beam, allowing their coupling to be measured. Taking $\mathrm{NEP}_{\mathrm{MB}}$ to refer to the main beam, we write

$$
\mathrm{NEFD}=\frac{\mathrm{NEP}_{\mathrm{MB}}}{\eta_{A} A_{\mathrm{tel}} \Delta \nu},
$$

where $\eta_{A}$, given in Eq. (2), distinguishes between a source filling the main beam and a true point source, and the bandwidth $\Delta v$ is the instrumental bandwidth.

It is customary, however, to describe heterodyne sensitivities with temperature units. To compare SPIFI with a heterodyne system, we convert the NEFD into a system temperature $T_{\text {sys. }}$. The flux density sensitivity $S(\mathrm{rms})$ is related to the rms antenna temperature $T_{A}{ }^{*}(\mathrm{rms})$ through

$$
S(\mathrm{rms})=\frac{2 k_{B} T_{A}^{*}(\mathrm{rms})}{\lambda^{2}} \Omega_{\text {beam }} \frac{1}{\eta_{\mathrm{MB}}} .
$$

Here $T_{A}{ }^{*}(\mathrm{rms})$ is given by the radiometer equation

$$
T_{A}^{*}(\mathrm{rms})=\frac{2 T_{\mathrm{sys}} \kappa}{\left(\Delta \nu t_{\mathrm{int}}\right)^{1 / 2}},
$$

where the 2 accounts for chopping and $\kappa$ is the backend degradation factor equal to 1.15 for correlator spectrometers. ${ }^{36}$ Because $S(\mathrm{rms})$ and the NEFD are essentially the same, $S(\mathrm{rms})=\mathrm{NEFD} / \sqrt{2 t_{\text {int }}}$ from Eq. (3), we have

$$
T_{\mathrm{sys}}=\frac{\mathrm{NEP}_{\mathrm{MB} \eta \mathrm{MB}}}{4 \sqrt{2} k_{B} \mathrm{\kappa} \sqrt{\Delta v}} .
$$

Unlike the NEP and NEFD, the system and rms antenna temperatures $\left(T_{\text {sys }}, T_{A}{ }^{*}\right)$ refer to the total coupling to the sky, i.e., the total efficiency that is not coupled to the ground, the dome, and supports. Thus the main beam efficiency is included in Eq. (7).

The values of the NEP, NEFD, and $T_{\text {sys }}$ for SPIFI on the JCMT are presented in Table 3, tabulated for several resolving powers. Sensitivities at $R=4800$ and $R=1500$ were measured at the telescope, although under poor atmospheric conditions of 5-10\% zenith transmission. Values presented are scaled to the conditions of a good night at zenith, $40 \%$ transmission, by means of simply scaling the observed SNR with sky transmission. We estimated the sensitivities at other resolving powers by combining the photon noise with the excess voltage noise and optical responsivity:

$$
\mathrm{NEP}^{2}=\mathrm{NEP}_{\text {photon }}{ }^{2}+\left(\frac{\mathrm{NEV}_{\text {excess }}}{S_{\text {opt }}}\right)^{2},
$$


Table 3. SPIFI Sensitivities at the $\mathrm{JCMT}^{a}$

\begin{tabular}{rccccc}
\hline \multicolumn{1}{c}{$R$} & $\begin{array}{c}\mathrm{NEP}^{b} \\
(\nu / \Delta \nu)\end{array}$ & $\begin{array}{c}\mathrm{NEFD}^{b} \\
(\mathrm{~W} / \sqrt{\mathrm{Hz}})\end{array}$ & $\begin{array}{c}T_{\text {sys }}{ }^{c} \\
(\mathrm{~K})\end{array}$ & $\begin{array}{c}T_{\text {sys }} \\
\mathrm{PBGL}^{d} \\
(\mathrm{~K})\end{array}$ & $\begin{array}{c}\text { Total BW }^{e} \\
\left(\mathrm{~km} \mathrm{~s}^{-1}\right)\end{array}$ \\
\hline 10000 & $1.4 \times 10^{-14}$ & 196 & 3640 & 1450 & 400 \\
4800 & $1.7 \times 10^{-14}$ & 114 & 3060 & 1450 & 810 \\
3000 & $1.9 \times 10^{-14}$ & 80 & 2700 & 1450 & 1300 \\
1500 & $2.4 \times 10^{-14}$ & 50 & 2410 & 1450 & 2000 \\
800 & $3.1 \times 10^{-14}$ & 34 & 2280 & 1450 & 3100 \\
\hline
\end{tabular}

${ }^{a}$ Sensitivities at the JCMT are based on measured values at $R=$ 4800 and 1500 , scaled to good weather conditions. Scanning is not included; effective system temperatures including scanning are $\sqrt{n_{\text {res el }}}$ larger, where $n_{\text {res el }}$ is the number of spectral resolution elements in the scan.

${ }^{b}$ The NEP refers to the main beam above the atmosphere $(40 \%$ transmission). NEFD refers to a true point soure, also above the atmosphere. Because these values depend on the various beam efficiencies, they are strong functions of the antenna at the time of observations.

${ }^{c}$ System temperatures refer to the forward beam above the atmosphere.

${ }^{d}$ System temperature of a perfect background-limited (PBGL) spectrometer at $810 \mathrm{GHz}$ on the JCMT. This estimate assumes an instrument transmission of $25 \%$, detector quantum efficiency of $50 \%$, reimaging lenses with a transmission of $75 \%$, telescope forward coupling of $65 \%$, and atmospheric transmission of $40 \%$.

eTotal bandwidth (BW) available in a single spectral setup.

where the $\mathrm{NEP}_{\text {photon }}$ is calculated theoretically (see Section 5) and NEV is the noise-equivalent voltage. The excess noise and optical responsivity are functions of power on the detector and bias current and are estimated from our load curves and noise measurements. Because the $1 / f$ noise and the optical responsivity both increase with resolving power, the second term on the right-hand side of Eq. (8) varies slowly, less than a factor of 2 over the range of resolving powers listed.

\section{Photon Background Noise}

To evaluate the instrument's performance, it is instructive to compare the measured sensitivities with the fundamental limit imposed by the fluctuations in the photon arrival rates, the background limit. The uncertainty in power $\sigma^{2}$ that is due to the photon background after an integration time $t_{\text {int }}$ can be expressed as $^{37-39}$

$$
\begin{aligned}
\sigma^{2}= & \frac{1}{t_{\mathrm{int}}} \sum_{i} \int \mathrm{d} \nu(h v)^{2}\left[n_{i}(T, v) \epsilon_{i} \tau_{i} \eta\right] \\
& \times\left[n_{i}(T, \nu) \epsilon_{i} \tau_{i} \eta+1\right] .
\end{aligned}
$$

The sum is over all spatial and polarization photon modes $i$ that couple to the detector, each with total coupling given by the product of emissivity $\epsilon_{i}$, transmission $\tau_{i}$, and detector quantum efficiency $\eta$. The $n_{i}$ are the usual photon-mode occupation numbers, $n_{i}=\left[\exp \left(h v / k T_{i}\right)-1\right]^{-1}$. We consider only the noise generated by the ambient radiation outside the instrument, which couples only in a small bandwidth and with a well-defined throughput:

$$
\sigma^{2}=\frac{\left(h \nu_{0}\right)^{2}}{t_{\mathrm{int}}} N_{m} \Delta \nu\left(n_{a, \nu_{0}} \epsilon_{\mathrm{amb}} \tau \eta\right)\left(n_{a, \nu_{0}} \epsilon_{\mathrm{amb}} \tau \eta+1\right),
$$

where the frequency interval has been approximated because the fractional bandwidth is small, equal to $\pi / 2$ times the resolution bandwidth because of the Lorentzian line shape of the Fabry-Perot. Because SPIFI's optical train couples only a single spatial mode from ambient temperature to each detector, the number of modes $N_{m}$ is 2 , including both polarizations.

The emissivity of the ambient radiation $\epsilon_{\text {amb }}$ includes contributions from the sky, telescope, and reimaging lenses:

$\epsilon_{\mathrm{amb}}=\left(1-t_{\text {lens }}\right)+t_{\text {lens }}\left[\left(1-\eta_{F}\right)+\eta_{F}\left(1-t_{\text {sky }}\right)\right]$,

where $t_{\text {lens }}=0.75$ is the total transmission of two polyethylene reimaging lenses, $\eta_{F}$ is the total forward coupling of the telescope $(0.65$ at $800 \mathrm{GHz}$ on the JCMT), and $t_{\text {sky }}$ is the transmission of the sky. On a good night at Mauna Kea, the zenith sky transmission is $40 \%$, resulting in a total emissivity of the ambient radiation of $\epsilon_{\mathrm{amb}}=0.81$. Other factors in the expression are the mode occupation number $n_{\mathrm{amb}, v_{0}}$ for which we take $T_{\mathrm{amb}}=280 \mathrm{~K}$, the instrument transmission $\tau=0.25$, and the detector quantum efficiency $\eta=0.5$. Combined together, the product $\gamma=n_{a, \nu_{0}} \epsilon_{\mathrm{amb}} \tau \eta$ measures the degree to which photons are correlated at the detector. For $\gamma<1$, the photons are uncorrelated, $\sigma \sim \sqrt{\gamma}$, and the noise obeys Poisson statistics as with optical and infrared instruments. For $\gamma>1, \sigma \sim \gamma \sim P_{\text {tot }}$; the photons are completely correlated and the uncertainty scales as the total incident power as with a traditional radio receiver. SPIFI operates near the threshold between these two regimes, with $\gamma$ between 0.7 and 0.8 .

To convert the uncertainty $\sigma$ to a measured SNR, we note that when we chop between a source and the background, the effective on-source integration time is half of the total integration time and that the noise is $\sqrt{2}$ larger because the background and source both contribute independent noise. Thus we have a measured SNR of

$$
\mathrm{SNR}=\frac{P_{\mathrm{amb}}}{\sqrt{2} \sqrt{2} \sigma},
$$

and we define the photon background-limited NEP according to Eq. (3):

$$
\mathrm{NEP}_{\text {photon }}=\frac{P_{\mathrm{amb}} \sqrt{2 t_{\mathrm{int}}}}{\mathrm{SNR}}=2 \sqrt{2} \sigma(1 \mathrm{~s})\left(\frac{\mathrm{W}}{\sqrt{\mathrm{Hz}}}\right) .
$$

These values are referred to the detector and include the detector quantum efficiency. To refer the NEP to the sky, this value is simply divided by the total coupling between the detector and the sky, which under good atmospheric conditions is 0.5 (quantum 
efficiency) $\times 0.25$ (cryogenic instrument) $\times 0.75$ (reimaging lenses) $\times 0.65$ (telescope forward coupling) $\times 0.4$ (atmospheric transmission) $=0.024$. Calculating this photon-limited NEP for the perfect background-limited (PBGL) system, referring it to the sky, and converting it into a system temperature, we obtain $T_{\text {sys }}($ PBGL) $=1450 \mathrm{~K}$ (see Table 3$)$.

For any real system, the true photon noise contribution is greater than what would be calculated according to the above method. Because the photons are somewhat correlated in SPIFI, an increase in the ambient emissivity from 0.81 to $1(23 \%)$, as was the case when our measurements were made, results in an increase in the photon NEP by $17 \%$, yielding a $T_{\text {sys }}$ of $1700 \mathrm{~K}$. Furthermore, out-of-band leakage power from ambient temperature outside and in-band radiation from inside the instrument also couple to the detectors and contribute to the noise. In SPIFI, the portion of the spectrometer at $60 \mathrm{~K}$ contributes power with low emissivity, approximately $10 \%$, but with a larger bandwidth because it is not filtered by the HOFPI. Depending on the operating parameters related to the resolving power, namely, the HOFPI and LOFPI orders and finesses, this $60-\mathrm{K}$ radiation contributes between 15 and $50 \%$ of the total detector power and an additional 8-20\% of the background noise. When this portion is included, the true photon noise $T_{\text {sys }}$, including scanning for SPIFI, is around $1830-2040 \mathrm{~K}$, a large portion of what is observed, particularly at the lower resolving powers. As the resolving power increases, both the background power and the signal power on the detector decrease and the intrinsic detector noise becomes more important.

As shown in Table 3, the system is within a factor of 1.5-3 of $T_{\text {sys }}$ (PBGL), the background limit for a perfect spectrometer with 25\% transmission and 50\% detector quantum efficiency. To compare this directly with a heterodyne spectrometer, we convert the $T_{\text {sys }}$ into a $T_{\mathrm{Rx}}$ using the heterodyne conventions $^{36}$ :

$$
T_{\mathrm{sys}}=2 \frac{T_{\mathrm{RX}}(\mathrm{DSB})+\eta_{F}\left(1-\eta_{\mathrm{sky}}\right) T_{\mathrm{sky}}+\left(1-\eta_{F}\right) T}{\eta_{\mathrm{sky}} \eta_{F}},
$$

where $\eta_{F}$ and $T_{\text {tel }}$ are the total forward coupling and physical temperature of the telescope, taken to include the losses in the polyethylene lenses; $\eta_{\text {sky }}$ and $T_{\text {sky }}$ are the transmission and physical temperature of the sky; and $T_{\mathrm{RX}}$ (DSB) represents the double sideband receiver temperature. Our measured system temperatures over the range of resolving powers imply equivalent $T_{\mathrm{RX}}$ (DSB) in a range from 40 to $130 \mathrm{~K}$. The best reported $800-\mathrm{GHz}$ heterodyne receiver temperature to date is $205 \mathrm{~K},{ }^{40}$ which is somewhat higher.

Equation (14) illustrates the difference between heterodyne and direct-detection noise conventionsthe expression of noise in a direct-detection system (e.g., NEP, NEFD) includes contributions from all noise sources, including the photon background, whereas the receiver temperature $T_{\mathrm{Rx}}$ represents only the additional noise generated in the receiver. In the model of the perfect background-limited system outlined above (with reimaging lenses) for which the photon NEP results in a system temperature of $1450 \mathrm{~K}$, the sky and telescope terms $\eta_{F}\left(1-\eta_{\text {sky }}\right) T_{\text {sky }}$ $+\left(1-\eta_{F}\right) T_{\text {tel }}$ in Eq. (14) would sum to $0.82 \times 280 \mathrm{~K}$ [see Eq. (11)]. The equivalent DSB receiver temperature is a nonsensical $-84 \mathrm{~K}$. The negative receiver temperature is due to the fact that the heterodyne conventions account for background and receiver noise coupling into both heterodyne sidebands, not appropriate for a direct-detection spectrometer. Without the factor of 2 in Eq. (14), the receiver temperature based on the photon-limited NEP is $+57 \mathrm{~K}$, greater than zero because the Fabry-Perot couples a larger noise bandwidth than the resolution bandwidth to which the sensitivity is referred.

Because SPIFI is a Fabry-Perot system, we must scan a few resolution elements to produce a spectrum; this raises the effective system temperature by $\sqrt{n}$. For a five-element scan, the factor of just over 2 in the system temperature results in a substantial change in the effective receiver temperature, yielding $T_{\mathrm{RX}}$ (DSB) ranging from 270 to $570 \mathrm{~K}$. This points out the disadvantage of a Fabry-Perot system; it couples only a single resolution element of bandwidth at at time. A grating spectrometer, by contrast, can couple many resolution elements simultaneously. If background-limited, a grating would be the most sensitive spectrometer possible for point sources. For observations of extended sources, however, a FabryPerot spectrometer such as SPIFI provides instantaneous two-dimensional mapping capability, which offsets the disadvantage of the single instantaneous spectral element.

We are indebted to S. H. Moseley and C. A. Allen at NASA Goddard Space Flight Center for providing SPIFI's bolometers. We thank several undergraduate students at Cornell University for their diligent efforts. Chuck Henderson, Wayne Holland, and the JCMT staff and operators deserve credit for their help interfacing SPIFI with the telescope, and we acknowledge Antony Stark and Peter Hargrave for their support during our first observing run. Finally, we thank an anonymous referee for several helpful suggestions. This research was made possible with NASA grants NAGW-4503 and NAGW-3925 and National Science Foundation grants OPP8920223 and OPP-0085812.

\section{References}

1. J. Kooi, Caltech Submillimeter Astrophysics, Pasadena, Calif. 91125 (personal communication, 2000).

2. R. Hills, J. Richer, S. Withington, H. Smith, H. Gibson, B. Dent, W. Duncan, J. Harris, P. Hastings, L. Avery, C. Cunningham, P. Feldman, R. Redman, K. Yeung, and P. Jewell, "Heterodyne array receiver programme for the James Clerk Maxwell Telescope" (2000), http://www.jach.hawaii.edu/ JACpublic/JCMT/Heterodyne_observing/Instrument_homes/ harp-info.html.

3. J. Keene, G. A. Blake, T. G. Phillips, P. J. Huggins, and C. A. 
Beichman, "The abundance of atomic carbon near the ionization fronts in M17 and S140," Astrophys. J. 299, 967-980 (1985).

4. J. Zmuidzinas, A. L. Betz, R. T. Boreiko, and D. M. Goldhaber, "Neutral atomic carbon in dense molecular clouds," Astrophys. J. 335, 774-785 (1988).

5. C. K. Walker, G. Narayanan, T. Buttgenbach, J. Carlstrom, J. Keene, and T. G. Phillips, "The detection of [CI] in molecular outflows associated with young stellar objects," Astrophys. J. 415, 672-679 (1993).

6. R. Plume, D. T. Jaffe, and J. Keene, "Observations of largescale [CI] emission from S140," Astrophys. J. 425, L49-L52 (1994).

7. W. Wild, A. I. Harris, A. Eckhart, U. U. Graf, J. M. Jackson, A. P. G. Russell, and J. Stutzki, "A multi-line study of the molecular interstellar medium in M 82's starburst nucleus," Astron. Astrophys. 265, 447-464 (1992).

8. A. I. Harris, "Directions for submillimeter and far-infrared instrumentation," in The Physics and Chemistry of the Interstellar Medium, Proceedings of the Third Cologne-Zermatt Symposium, V. Ossenkopf, J. Stutzki, and G. Winnewisser, eds. (GCA-Verlag Herdecke, Germany, 1999).

9. J. E. Carlstrom and J. Zmuidzinas, "Millimeter and submillimeter techniques," in Reviews Radio Science 1993-1995, W. R. Stone, ed. (Oxford U. Press, Oxford, UK, 1996).

10. J. R. Tucker and M. J. Feldman, "Quantum detection at millimeter wavelengths," Rev. Mod. Phys. 57, 1055-1113 (1985).

11. M. Gerin and T. G. Phillips, "Atomic carbon in Arp 220," Astrophys. J. 509, L17-L20 (1998).

12. G. J. Stacey, J. W. Beeman, E. E. Haller, N. Geis, A. Poglistch, and M. Rumitz, "Stressed and unstressed Ge:Ga photoconductor arrays for far-IR astronomy," Int. J. Infrared Millim. Waves 13, 1689-1700 (1991).

13. W. S. Holland, C. R. Cunningham, W. K. Gear, T. Jenness, K. Laidlaw, J. F. Lightfoot, and E. I. Robson, "SCUBA, a submillimeter camera operating on the James Clerk Maxwell Telescope," in Advanced Technology MMW, Radio, and Terahertz Telescopes, T. G. Phillips, ed., Proc. SPIE 3357, 305-318 (1998).

14. N. Wang, T. R. Hunter, D. J. Benford, E. Serabyn, D. C. Lis, T. G. Phillips, S. H. Moseley, K. Boyce, A. Szymkowiak, C. Allen, B. Mott, and J. Gygax, "Characterization of a submillimeter high-angular-resolution camera with a monolithic silicon bolometer array for the Caltech Submillimeter Observatory," Appl. Opt. 35, 6629-6640 (1996).

15. M. R. Swain, C. M. Bradford, G. J. Stacey, A. D. Bolatto, J. M. Jackson, M. Savage, and J. A. Davidson, "Design of the South Pole Imaging Fabry-Perot Interferometer (SPIFI), " in Infrared Astronomical Instrumentation, A. Fowler, ed., Proc. SPIE 3354, 480-492 (1998).

16. H. M. Latvakoski, "High spatial resolution mid and farinfrared imaging of the Galactic Center," Ph.D. dissertation (Cornell University, Ithaca, N.Y., 1997).

17. A. Poglitsch, J. W. Beeman, N. Geis, M. Haggerty, E. E. Haller, J. M. Jackson, M. Rumitz, G. J. Stacey, and C. H. Townes, "The MPE/UCB far-infrared imaging Fabry-Perot interferometer (FIFI)," Int. J. Infrared Millim. Waves 12, 859-870 (1991).

18. A. Harper, R. H. Hildebrand, R. Stiening, and R. Winston, "Heat trap: an optimized far infrared field optics system," Appl. Opt. 15, 53-60 (1976).

19. D. J. Schroeder, Astronomical Optics (Academic, San Diego, Calif., 1987), Chap. 15

20. M. Born and E. Wolf, Principles of Optics (Pergamon, Oxford, UK, 1980), Chap. 7.

21. G. J. Stacey, T. L. Hayward, H. M. Latvakoski, and G. E. Gull, "KWIC: a widefield mid-infrared array camera/spectrometer for the KAO," in Infrared Detectors and Instrumentation, A. M. Fowler, ed., Proc. SPIE 1946, 238-248 (1993).
22. K. Sakai and L. Genzel, "Far-infrared metal mesh filter and Fabry-Perot interferometry," in Reviews of Infrared and Millimeter Waves, K. J. Button, ed. (Plenum, New York, 1983).

23. D. McCammon, W. Cui, M. Juda, J. Morgenthaler, J. Zhang, R. L. Kelley, S. S. Holt, G. M. Madejski, S. H. Moseley, and A. E. Szymkowiak, "Thermal calorimeters for high resolution X-ray spectroscopy,” Nucl. Instrum. Methods Phys. Res. A 326, 157-165 (1993).

24. S. H. Moseley, J. C. Mather, and D. McCammon, "Thermal detectors as X-ray spectrometers,” J. Appl. Phys. 56, 12571262 (1984).

25. G. H. Rieke, Detection of Light from the Ultraviolet to the Submillimeter (Cambridge U. Cambridge, Mass., 1994), Chap. 9.

26. J. C. Mather, "Bolometers: ultimate sensitivity, optimization, and amplifier coupling," Appl. Opt. 23, 584-588 (1984).

27. S.-I. Han, R. Almy, E. Apodaca, W. Bergmann, S. Deiker, A. Lesser, D. McCammon, K. Rawlins, R. L. Kelley, S. H. Moseley, F. S. Porter, C. K. Stahle, and A. E. Szymkowiak, "Intrinsic 1/f noise in doped silicon thermistors for cryogenic calorimeters," in EUV, X-Ray and Gamma-Ray Instrumentation for Astronomy IX, O. H. Siegmund and M. A. Gummin, eds., Proc. SPIE 3445, 640-644 (1998).

28. G. K. White, Experimental Techniques in Low-Temperature Physics (Clarendon, Oxford, UK, 1979), Chap. 9.

29. H. E. Fischer, "Magnetic cooling," in Experimental Techniques in Condensed Matter Physics at Low Temperatures, R. C. Richardson and E. N. Smith, eds. (Addison-Wesley, Reading, Mass., 1998).

30. D. McCammon, R. Almy, S. Deiker, J. Morgenthaler, R. L. Kelley, F. J. Marshall, S. H. Moseley, C. K. Stahle, and A. E. Szymkowiak, "A sounding rocket payload for X-ray astronomy employing high-resolution microcalorimeters," Nucl. Instrum. Methods Phys. Res. A 370, 266-268 (1996).

31. J. Ashok, P. L. H. Varaprasad, and J. R. Birch, "Polyethylene," in Handbook of Optical Constants of Solids II, E. D. Palik, ed. (Academic, Boston, Mass., 1991).

32. R. Stark, Max-Planck-Institute für Radiastronomie, Bonn, Germany (personal communication, 2000).

33. See paper by R. Stark, "MPIRE: the MPIfR/SRON $800 \mathrm{GHz}$ heterodyne spectrometer," http://www.mpifr-bonn.mpg.de/ $\operatorname{div} / \mathrm{mm} /$ tech/mpire.html, (2000).

34. J. R. Pardo, J. Cernicharo, and E. Serabyn, "Atmospheric transmission at microwaves (ATM): an improved model for mm/submm applications," IEEE Trans. Antennas Propag. 49, 1683-1694 (2001).

35. J. R. Pardo, J. Cernicharo, and E. Serabyn, "Submillimeter atmospheric transmission measurements on Mauna Kea during extremely dry El Nino conditions: implications for broadband opacity contributions," J. Quant. Spectrosc. Radiat. Transfer 68, 419-433 (2001).

36. See paper by H. Matthews, "Estimating time requirements and sensitivity for heterodyne receivers" (1999), http://www. jach.hawaii.edu/JACpublic/JCMT/User_documentation/ Users_guide/guide/node29.html.

37. J. M. Lamarre, "Photon noise in photometric instruments at far-infrared and submillimeter wavelengths," Appl. Opt. 25, 870-876 (1986)

38. J. Zmuidzinas, Caltech Submillimeter Astrophysics, Pasadena, Calif. (personal communication (2000).

39. D. J. Benford, T. R. Hunter, and T. G. Phillips, "Noise equivalent power of background limited thermal detectors at submillimeter wavelengths," Int. J. Infrared Millim. Waves 19, 931-938 (1998).

40. J. W. Kooi, J. Kawamura, J. Chen, G. Chattopadhyay, J. R. Pardo, J. Zmuidzinas, T. G. Phillips, B. Bumble, J. Stern, and H. G. LeDuc, "A low-noise, NbTiN-based $850 \mathrm{GHz}$ SIS receiver for the Caltech Submillimeter Observatory," Int. J. Infrared Millim. Waves 21, 1357-1373 (2000). 